\title{
Design of Smart Southwest Tourism Monitoring and Management System
}

\author{
Xuejie Hao ${ }^{1}$, Xiuhong $\mathrm{Li}^{1}$, Lizeyan Yin ${ }^{2}$, Le Zhang ${ }^{3}$, Rongjin Yang ${ }^{3+}$, Qiang Liu ${ }^{1}$ and Meiying \\ Sun ${ }^{1}$ \\ ${ }^{1}$ State Key Laboratory of Remote Sensing Science, College of Global Change and Earth System Science, \\ Beijing Normal University, No.19, Xinjiekou Wai Street, Haidian District, Beijing 100875, China; \\ ${ }^{2}$ University Clermont Auvergne, INP, Higher Institute of Computer Modeling and their Applications, \\ ISIMA, Aubiere Cedex, France; \\ ${ }^{3}$ Chinese Research Academy of Environmental Sciences, No.8, Da Yang Fang, An Wai, Chao Yang, Beijing \\ 100012, China;
}

\begin{abstract}
With the rapid economic development, the improvement of material and cultural levels, and the transformation of people's consumption consciousness, the tourism industry has shown full of vitality, especially in the southwestern region with lucid water and lush mountains, and primitive landscapes have become a tourist hotspot. On the one hand, there is a surge in tourists. Another aspect is the protection of the environment. How to better promote the development of tourism in the protection, so that both have a harmonious development, it is necessary to invest heavily in management. This paper designed a smart southwest tourism monitoring and management system based on wireless sensors for the southwest tourism area. This system changed the design of the previous monitoring system that focused on pollutant monitoring but instead monitors the beautiful environmental quality parameters such as air quality (PM2.5, negative oxygen ions, etc.) and the ecological environment that can reflect the original characteristics of the Southwest. At the same time, it can also conduct real-time monitoring and early warning of pest control, human activities, and fire conditions. The system can collect, transmit, display, store and analyze environmental indicators in real-time. It has the advantages of low construction cost, flexible and convenient layout, and high precision, which can meet the current needs of building tourism area monitoring and management.
\end{abstract}

Keywords: Wireless sensor network, Southwest tourist area of China, Air quality monitoring, Ecological environment monitoring

\section{Introduction}

With the development of the economy, the tourism industry has also developed vigorously and has become one of the industries with the strongest momentum and the largest scale in the development of the national economy. The 21st century is the information age. With the popularization of modern communication technology in various fields of economic life, tourism informatization has become a barometer for the decision-making of tourism departments. The use of modern monitoring and management systems in tourist attractions is an inevitable trend of current development [1].

Monitoring standards for tourist attractions are as follows: (1) The monitoring object is comprehensive monitoring that includes humans and nature. There are tourist activities in the tourist area, and these activities will have an impact on the ecological environment of the tourist area. Therefore, the monitoring of the humanistic system including tourists is essential. (2) The monitoring area is the entire carrier of the tourist area. Tourist areas mostly depend on areas such as nature reserves, forest parks, geological parks, and undeveloped mountains and rivers. These primitive areas protected by national laws should be strictly functionally zoned during eco-tourism planning, and planned for rational use. (3) It is necessary to make full use of various monitoring methods, such as the use of weather stations and environmental monitoring stations outside the eco-tourism area to collect relevant data. (4) The monitoring and management system of

\footnotetext{
+ Corresponding author. Tel.: +86 13621136123; fax: None

E-mail address: yangrj@craes.org.cn
} 
the tourist area can not only complete the basic ecological environment monitoring tasks but also provide an effective guarantee for the sustainable development of eco-tourism [2].

Based on the tourism ecological monitoring system, there have been some research foundations at home and abroad. In 2007, Wu Jinying designed and implemented a pedestrian flow monitoring and statistics system in tourist areas. The system is mainly based on the XScale series processor chip PXA270 as the core of the hardware system, transplanting embedded Linux as the operating system, and QtE as the embedded graphical interface, and developing corresponding human flow detection and tracking programs, video compression programs, short message sending and receiving programs, etc. The system finally realizes the monitoring and statistics of the flow of people in the tourist area, personnel scheduling, etc., which improves the efficiency of tourism management, and at the same time provides accurate and fast tourism information for tourists [3]. In 2012, Wang Xiaofang took the water of the Yabuli Ski Resort as the research area, carried out ecological monitoring of the water source in the area, and made a health evaluation [4]. In 2014, Shi Weifeng used the Chongming Scenic Tourism Area as the research area to monitor the negative oxygen ions in the area and analyzed the factors affecting negative oxygen ions [5]. In 2017, Chen Jiaxun, Yu Ting et al. conducted an aquatic organism survey and monitoring to evaluate the water quality safety of the Yabuli water source and further analyzed the impact of project expansion on the water quality and aquatic organisms of Yabuli water source through biological monitoring [6]. In 2018, Lan Meiling designed a set of an ecological environment monitoring system, using the Internet of Things technology, sensor principles, and GIS technology to collect and dynamically display the changes in the Lushan ecological environment in real-time, combining static and historical socio-economic indicator data and dynamic real-time ecological environment The combination of index data can more rationally evaluate the ecological health of tourist attractions and the impact of human activities on the ecological environment [7]. In 2019, Lin et al. developed remote sensing technology to monitor changes in forest resources. The technology has rapid characteristics and real-time spatial information collection and analysis capabilities. It is concluded that dynamic monitoring can effectively monitor and protects forest vegetation, which provided ideas for the follow-up forestry planning and ecological tourism development in Zhangjiakou [8]. Based on the current research status, the monitoring systems in tourist areas have the disadvantages of single monitoring objects and limited application, which cannot meet the need for comprehensive monitoring and wide coverage in tourist areas.

To meet the needs of tourist area monitoring, this paper designs a southwest tourist area monitoring system based on a wireless sensor network. The goal is to build a low-cost, comprehensive monitoring, widecoverage, and reusable monitoring system to meet the needs of tourists for real-time mastery of tourist area data.

\section{Overview of the Construction Area}

This paper takes the southwest region under the natural division as the layout area $\left(20^{\circ} 54^{\prime} \mathrm{N} 34^{\circ}\right.$ $19^{\prime} \mathrm{N}, 91^{\circ} 21^{\prime} \mathrm{E} 112^{\circ} 04^{\prime} \mathrm{E}$ ). It is located in the vast hinterland of the western part of southern China (excluding the Qinghai-Tibet Plateau), and mainly includes topographic units such as the Sichuan Basin, Qinba Mountains, and Yunnan-Guizhou Plateau. It roughly includes eastern Sichuan Province, Chongqing, southern Shaanxi Province, most of Yunnan Province, Guizhou Province, western Hubei Province, western Hunan Province, and northwest Guangxi. The southwest region in this study includes 1 municipality directly under the central government, 14 autonomous prefectures, and 46 prefecture-level cities. The land area of the five provinces, autonomous regions, and municipalities in the southwest region reached 1,375,200 $\mathrm{km}^{2}$, accounting for approximately $14.33 \%$ of the Chinese land area. The location information is shown in Fig. 1.

The southwest region includes the three major steps of Chinese topography, with large undulations and complex topography. It mainly includes the Western Sichuan Plateau, the Yunnan-Guizhou Plateau, the Hengduan Mountains, the Guangxi hills, and the Sichuan Basin. The topographic map of the southwest region is shown in Fig. 2. Corresponding to the terrain area, the climate types in the southwestern region are diverse, mainly including alpine cold zone climate, temperate monsoon climate, and subtropical monsoon climate. Northwest to southeast, the temperature and precipitation are very different, and the temporal and spatial distribution is extremely uneven. Southwest China is rich in water resources. The southwestern region 
has relatively developed water systems and densely distributed river networks, including the Yangtze River, the Yellow River, the Nu River, the Lancang River, the Pearl River, the Irrawaddy River, and the Yuanjiang River. The water collection area is large and it is the most important water resource enrichment area in China [9]. The precipitation in the southwest is relatively sufficient, but the distribution is quite different. In terms of time, about $80 \%$ to $90 \%$ of the annual precipitation are concentrated in May to October, and only about $10 \%$ to $20 \%$ of the precipitation are distributed from November to April the following year. Spatially, precipitation varies between $600-2300 \mathrm{~mm}$ from northwest to southeast, generally showing a distribution pattern of decreasing from southeast to northwest. The biological resources in the southwest region are rich and diverse. The southwest region is one of the 35 hotspots of biodiversity distribution in the world and a key area of global biodiversity. The number of nationally protected species in the southwestern region is 392, accounting for $66 \%$ of the national total. The southwest region is rich in forest resources. In 2018, the average forest coverage rate of the 5 provinces, autonomous regions, and municipalities in Southwest China reached $53.36 \%$, which was $30.4 \%$ higher than the Chinese forest coverage rate of $22.96 \%$. Among them, forest coverage rates of Sichuan, Chongqing, Guizhou, Yunnan, and Guangxi are 38.83\%, 48.3\%, 57\%, $60.3 \%$, and $62.37 \%$. The Southwest Forest Region is the second-largest natural forest area in China. The total forest stock in this area has reached $21.5 \times 108 \mathrm{~m}^{3}$, which accounts for about $25 \%$ of Chinese total forest stocks [10-11].

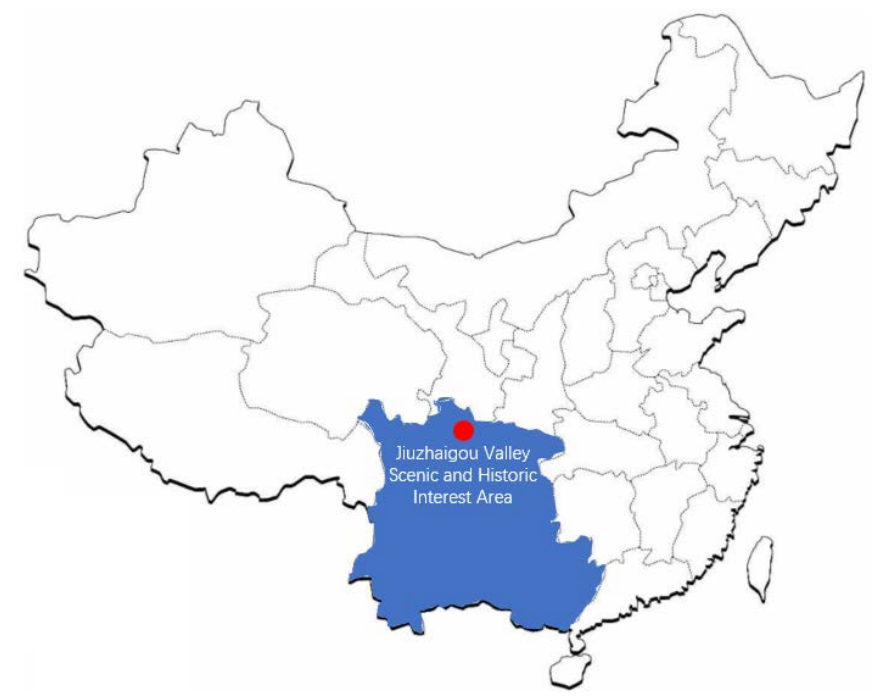

Fig. 1: Location of the southwest tourist area.

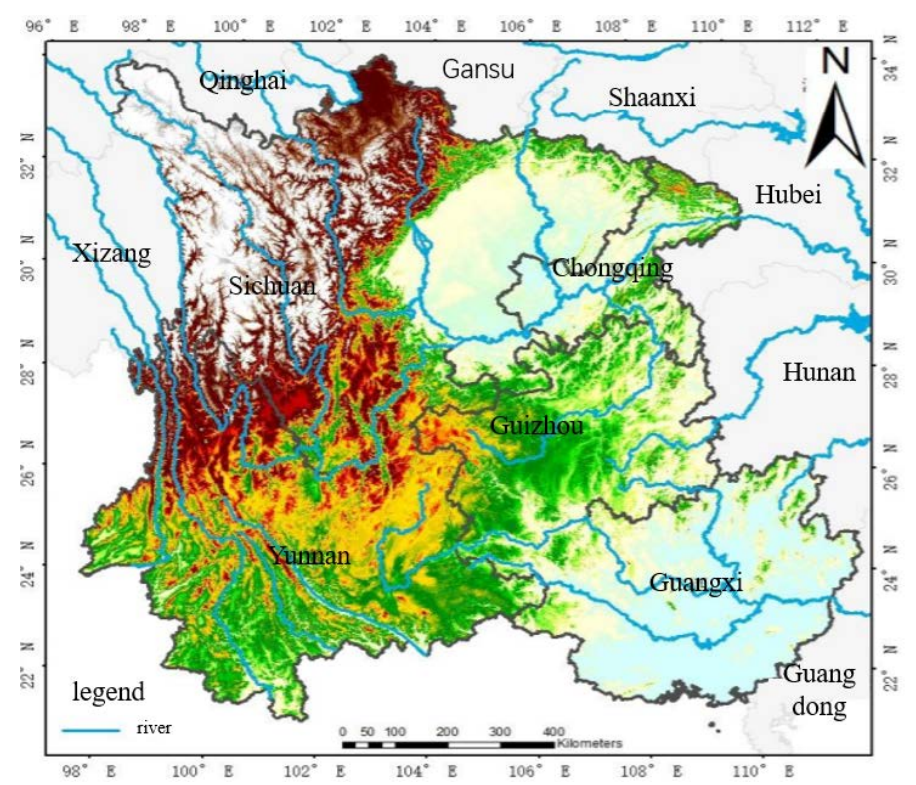

Fig. 2: Topographic map of Southwest China 
This article selects the famous tourist attraction "Jiuzhaigou National Nature Reserve" in the southwest tourist area for experiments (the location is shown in Fig. 1). Jiuzhaigou is located in Jiuzhaigou County, Aba Tibetan, and Qiang Autonomous Prefecture, Sichuan Province. Located on the Qinghai-Tibet Plateau, Western Sichuan Plateau, and the transition zone from mountain to Sichuan Basin, it is more than 300 kilometers south of Chengdu. It is a mountain valley with a depth of more than 50 kilometers, with a total area of 64297 hectares and a forest coverage rate of over $80 \%$. There are 74 nationally protected rare plants, 18 nationally protected animals, and rich paleontological fossils and ancient glacier landforms in the reserve. Jiuzhaigou National Nature Reserve is the core area and corridor belt of the giant panda A population in the Minshan Mountains. It has a typical natural ecosystem and is one of the cores of biodiversity protection in China. Rich in animal and plant resources, it has extremely high ecological protection, scientific research, and aesthetic tourism value. The biodiversity of the region is rich, and the rare species are outstanding. Jiuzhaigou is also known as "Six Uniqueness of Jiuzhaigou" based on the mountains and lakes, waterfalls, forests, snow-peaks, ice, and Tibetan customs. It is known as the "Fairy Tale World" and the "King of Water Scenery" by the world. Jiuzhaigou is also a national geopark with geological heritage calcified lakes, beach streams, waterfall landscapes, karst water systems, and forest ecosystems as the main protection objects, and has extremely high scientific research value [12].

\section{Monitoring Management System Design}

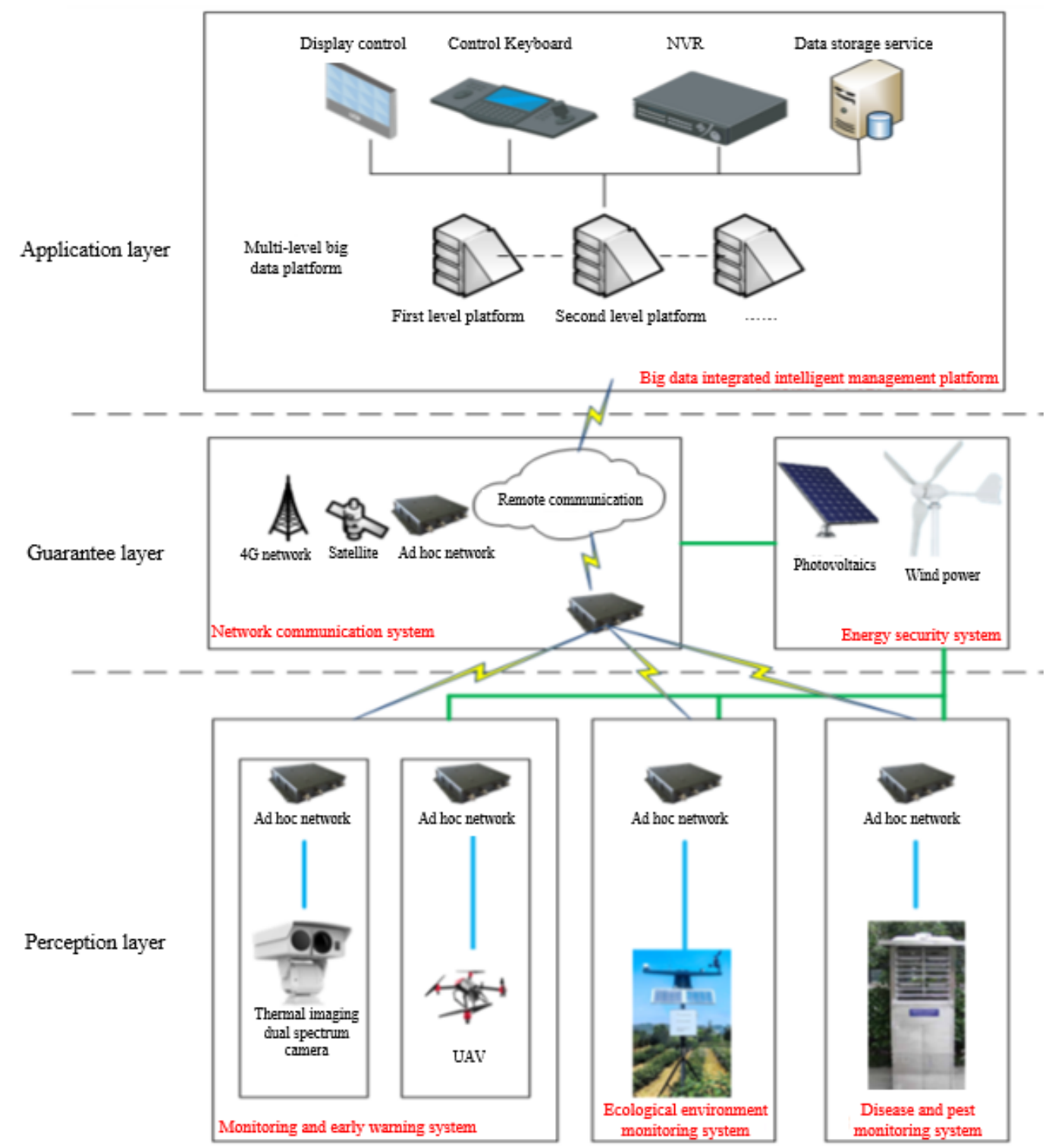

Fig. 3: The framework of the monitoring and management system in the southwest tourist area. 
According to the characteristics of the southwest tourist area, this paper designs a monitoring and management system for the southwest smart tourist area. Its system framework is shown as in Fig. 3. The system architecture can be divided into three levels: The perception layer, the guarantee layer, and the application layer. The system is mainly composed of six subsystems: Network communication subsystem, monitoring, and early warning subsystem, energy security subsystem, ecological environment monitoring subsystem, pest monitoring subsystem, and big data integrated intelligent management platform.

Technical characteristics of the system: Real-time video, danger signal, disaster monitoring, and identification; Intelligent key area monitoring and disaster prevention and control; Accurate location of dangerous and disaster areas and personnel positions; High-bandwidth wireless ad hoc network coverage and heterogeneous massive data remote transmission; Realize the energy self-sufficiency of the equipment in the monitoring area through wind-solar complementation; The super-large screen of the command center is centrally displayed (the screen can be split), and the back-end big data analysis system can intelligently identify the monitoring data.

The advantages of the system are (1) Wide coverage monitoring: Using dual-spectrum thermal imaging cameras, using the most advanced uncooled infrared technology and optical imaging technology, to achieve 24-hour unmanned day and night continuous monitoring. (2) No need for communication network support: This system uses wireless ad hoc network equipment to complete the deployment and control of network communication transmission in the tourist area without the support of public network operators. (3) High early warning and response capabilities: The method of "stay detection in the visible light field one by one" is adopted to realize automatic detection-visible light detection of smoke and infrared thermal imaging detection of fire points to ensure timely detection of fires. (4) Strong scalability: Fully consider the needs of tourist area monitoring and the system expansion brought about by future technological development, and reserve enough interface space to meet the requirements of software upgrades, equipment expansion, and functional expansion. (5) No need for local power grid support: By setting up a small wind-solar hybrid power generation system, equipped with a battery energy storage system. Can provide 7-10 rain and windless days with a power supply. (6) Strong environmental adaptability: Not affected by rain, snow, fog, haze, strong light, backlight, low light, and no light.

\section{Key Technology}

\subsection{Wireless ad hoc Network Technology}

Ad hoc network is a multi-hop peer-to-peer network formed by several communication nodes with equal status through dynamic self-organization [13-14]. The technology originated from the United States DARPA (US Department of Defense Advanced Research Projects Agency). It has always been an important military network technology. In recent years, with the progress of wireless communication technology and the development of the military-civilian integration industry, it has gradually expanded to the civilian field. Jiangsu Zhongli Electronics adopts independent research and development of channel aggregation technology, selects the best frame aggregation method and MAC access method, and uses the physical layer super needle technology to aggregate and transmit data frames sent to different nodes, which is a good solution to the node Problems such as density, transmission distance, Doppler shift and spatial fading caused by changes in communication links have realized the long-distance transmission of high-bandwidth wireless ad hoc networks. Due to the characteristics of dynamic ad hoc networks, the devices in ad hoc networks are generally wireless devices, and the networks formed by them are usually also called wireless ad hoc networks. The ad hoc network has the characteristics of no center, each node in the network has equal status, and usually integrates the functions of transmitting and receiving and playing multiple roles such as a terminal, relay, and routing at the same time. Because of this feature, the construction of the ad hoc network does not require fixed facilities at all and has a strong survivability. The wireless self-organizing network is especially suitable for field operations in tourist areas, mines, archaeology, and prospecting where there is no or inconvenient setting up a wired network.

The advantages of the self-organizing network connection method are: Point-to-point direct connection or multi-hop connection can be realized between terminals, and any node has a relay and forwarding function; 
The communication link between terminals changes dynamically; Can form a network without relying on any fixed facilities. The specific connection method is shown in Fig. 4.

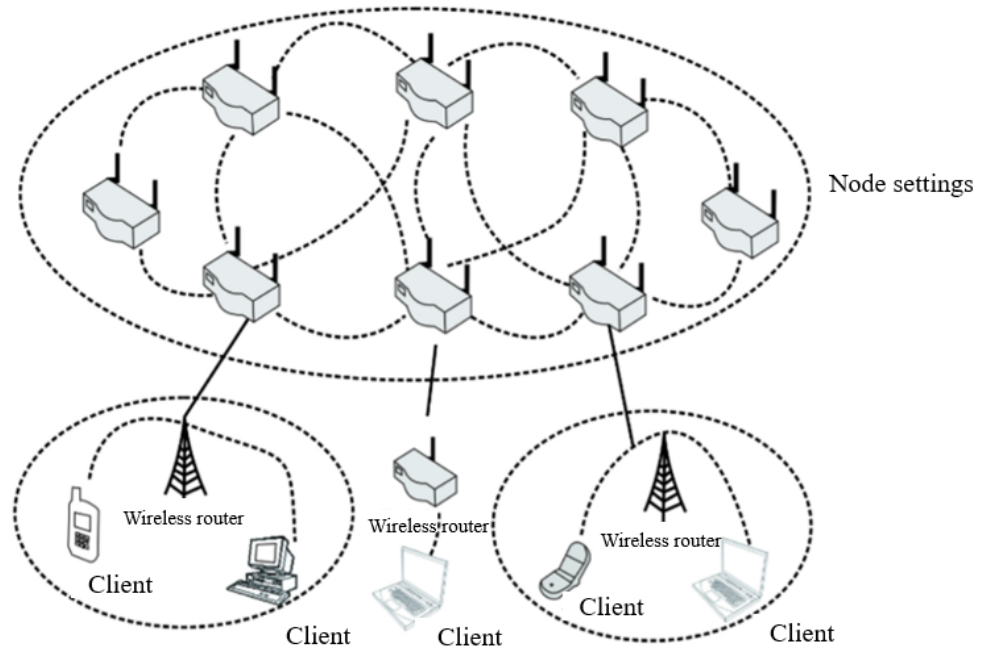

Fig. 4: Ad hoc network connection mode.

Wireless ad hoc network technology has the characteristics of network self-organization, network selfhealing, and multi-hop connection that can realize non-line-of-sight transmission. Network self-organization: the network is formed automatically, and the best business path can be calculated in real-time without manual intervention. Network self-healing: when the node equipment is damaged, the network quickly selfheals to ensure real-time communication. The multi-hop connection can realize non-line-of-sight transmission: the self-organizing network can effectively bypass obstacles and ensure network connection by reasonably setting intermediate nodes.

\subsection{Network Communication Subsystem}

The network communication subsystem uses wireless ad hoc network communication equipment to complete the construction of the communication network in the tourist area; Adopt wireless ad hoc network combined with traditional satellite, $4 \mathrm{G}$, microwave, or optical fiber to complete the network communication between the front monitoring center point and the rear monitoring command center [15-16]. The framework of the network communication subsystem is shown in Fig. 5.

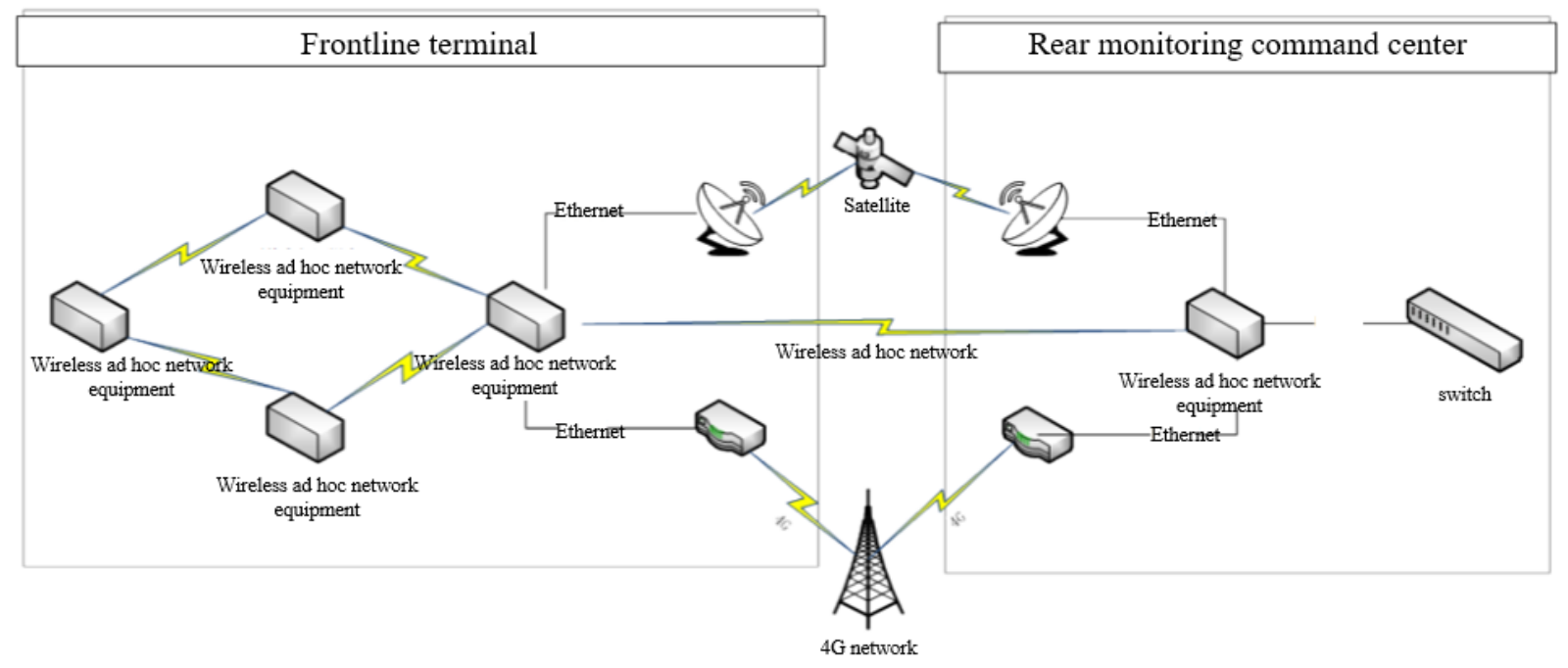

Fig. 5: Network communication subsystem framework.

The network communication subsystem is mainly divided into two parts: the communication in the tourist area and the remote communication. Tourist area communication: In the tourist area, 4 terminal points and 1 central point are arranged, and wireless ad hoc network equipment is used to complete the network. The information of each point in the tourist area is gathered at the central point and transmitted to the 
monitoring and command center at the back through the remote communication subsystem. Remote communication subsystem: It is composed of wireless ad hoc network equipment as the main body. According to the actual situation, to meet the transmission needs of multi-channel high-definition real-time video information, different methods such as self-organizing network chain jumper, 4G communication, optical fiber communication, microwave communication, satellite communication, etc. can be used.

\subsection{Monitoring and Early Warning Subsystem}

The monitoring and early warning subsystem use thermal imaging dual-spectrum cameras for conventional 24-hour unattended monitoring, and UAV (Unmanned Aerial Vehicle) for inspection and emergency monitoring to establish a three-dimensional integrated networked joint control image resource library and an integrated image monitoring application system [17-18]. The framework of monitoring and early warning subsystem is shown in Fig. 6. The thermal imaging dual-spectrum camera uses high-sensitivity infrared detectors, infrared lenses, visible light lenses, high-definition cameras, and intelligent variable-speed pan/tilt. Using the most advanced uncooled infrared technology and optical imaging technology, it is assembled through scientific and advanced technical means. Visible light can display high-definition color images during the day, and infrared thermal imaging can provide sharp and clear thermal images, realizing continuous monitoring day and night in a 24-hour all-weather (no light/backlight/strong light, rain/snow/fog) environment; When a suspected fire is found, the UAV will be used to fly to the scene for retesting to ensure that there is no blind spot and full coverage of the video surveillance area in the tourist area.

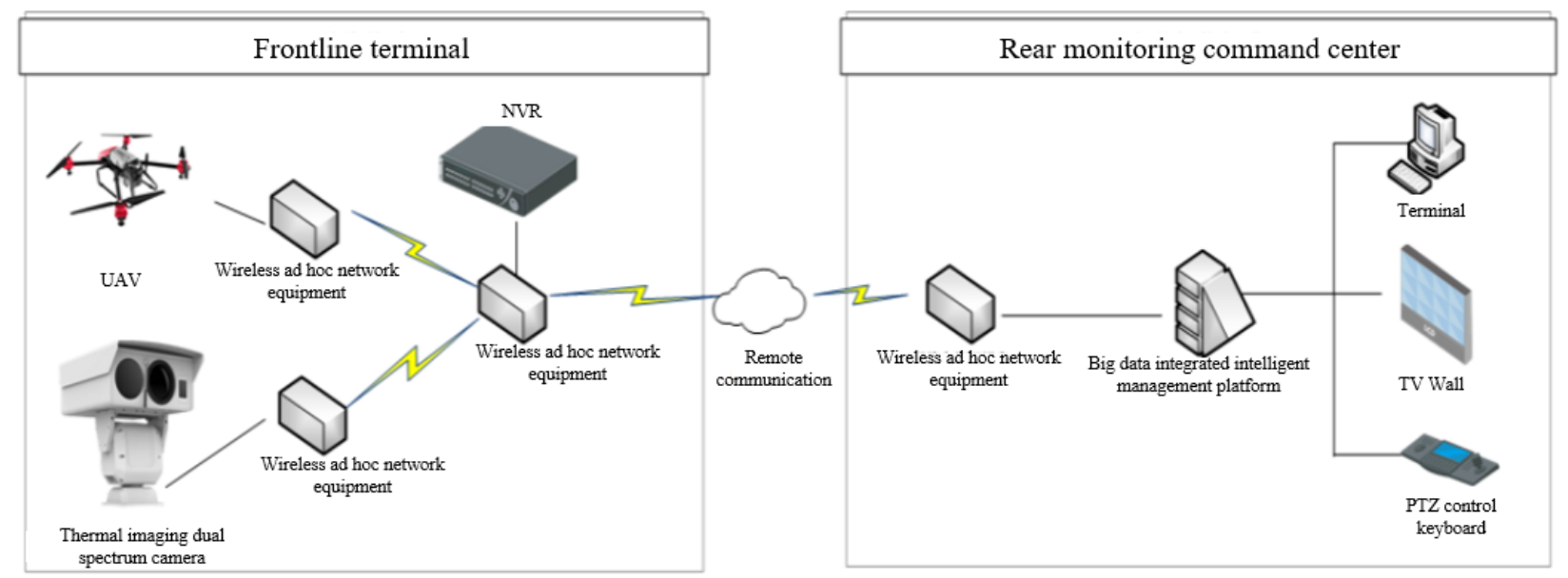

Fig. 6: Monitoring and early warning subsystem framework.

Monitoring and early warning subsystem is composed of monitoring and early warning equipment, storage equipment, and display control equipment. Set up a dual-spectrum thermal imaging camera in the tourist area, you can choose the ZGX-CT series dual-spectrum remote thermal imaging camera of China Optics Group Corporation. Its advantage is that it can penetrate dust, smoke, rain, snow, and darkness, and realize remote video surveillance all day and night. The collected video data is gathered to the central point through the wireless ad hoc network, and then the data is transmitted to the command center located at the rear through the remote communication subsystem. The subsystem has functions: (1) Intelligent analysis and recognition function: Infrared thermal imaging cameras can accurately find high-temperature objects. By setting automatic monitoring paths and plans, automatic fire identification and alarms can be realized. Under the premise of ensuring the detection speed, the method of "visible light field-by-field stay detection" is adopted to detect smoke with visible light and fire with infrared to realize automatic detection to ensure early detection of fire. (2) Fog penetration function: Adopting MIT imaging technology, integrated optical fog penetration, SSD fog penetration algorithm, and adaptive fog penetration trinity, which can cover all-around imaging of different wavelength light sources. The self-adaptive fog penetration algorithm can realize automatic switching of different modes between scenes, ensuring all-weather monitoring and presenting a strong fog penetration effect at all times. (3) Storage function: This subsystem adopts NVR centralized storage architecture with a storage time of 30 days, and all images are stored in high-definition 1080P format. The use of streaming storage to improve system reliability and performance and the use of advanced high- 
definition low-bit stream cameras can meet the storage needs of a single high-definition 1080P $(1920 \times 1080)$ cameras for 30 days.

\subsection{Energy Security Subsystem}

Due to the lack of effective infrastructure in tourist areas, combined with the analysis of local meteorological conditions, the use of wind and solar hybrid power generation systems to ensure energy needs [19]. The framework of the energy security subsystem is shown in Fig. 7. Specific plan: The windsolar complementary approach is adopted, and a wind-solar complementary small power generation and energy storage system are installed at each node. Power supply capacity: The battery pack of each node is fully charged and can supply power for 7-10 days. Charging time: Pre-charge for 5 days (when the system is not working); When the monitoring system is working normally, it takes 5 to 25 days to fully charge the battery. System maintenance: Due to the normal wear and tear of the battery pack, to ensure normal use, it is recommended to maintain and replace the battery pack every 3 years.

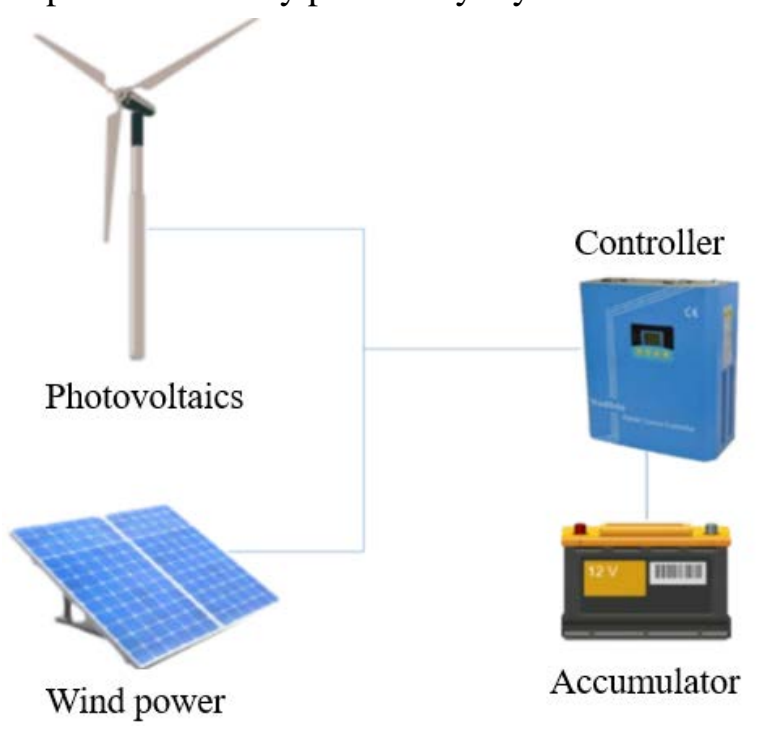

Fig. 7: Energy security subsystem framework.

Photovoltaic power generation equipment adopts Tenghui Photovoltaic's polycrystalline solar photovoltaic modules. It has the following characteristics: advanced half-cell battery technology, industryleading power generation efficiency gain, excellent resistance to potential induced attenuation (PID) performance, high impermeability, and strong wear resistance. It can be widely used in harsh environments such as high humidity and strong wind and sand.

\subsection{Ecological Environmental Monitoring Subsystem}

Table 1: Data parameters collected by various sensors in the ecological environment monitoring subsystem

\begin{tabular}{|l|l|l|l|}
\hline Sensor & Measuring range & Resolution & Accuracy \\
\hline Soil moisture & $0 \sim 100 \%$ & $0.1 \%$ & $\pm 0.3 \%$ \\
\hline Soil temperature & $-40 \sim 100^{\circ} \mathrm{C}$ & $0.1^{\circ} \mathrm{C}$ & $\pm 0.5^{\circ} \mathrm{C}$ \\
\hline Air temperature & $-40 \sim+120^{\circ} \mathrm{C}$ & $0.1^{\circ} \mathrm{C}$ & $\pm 0.4^{\circ} \mathrm{C}$ \\
\hline Air humidity & $0 \sim 100 \%$ & $0.1 \%$ & $\pm 3 \% \mathrm{RH}$ \\
\hline Radiation & $0 \sim 2000 \mathrm{~W} / \mathrm{m}$ & $1 \mathrm{~W} / \mathrm{m}$ & $\pm 1 \mathrm{~W} / \mathrm{m}$ \\
\hline Wind direction & $0 \sim 359^{\circ}$ & $1^{\circ}$ & $\pm 1^{\circ}$ \\
\hline Wind speed & $0 \sim 45 \mathrm{~m} / \mathrm{s}$ & $\pm 0.1 \mathrm{~m} / \mathrm{s}$ & $\pm(0.3+0.03 \mathrm{v}) \mathrm{m} / \mathrm{s}$ \\
\hline Precipitation & $0 \sim 6553 \mathrm{~mm}$ & $0.1 \mathrm{~mm}$ & $\pm 0.1 \mathrm{~mm}$ \\
\hline
\end{tabular}

The ecological environment monitoring subsystem collects the weather, soil, hydrology, water quality, air quality, and other elements of the tourist area in real-time through various sensors set up on the site (as shown in Table 1). Through network transmission and system transmission to the rear command center, the entire system can realize automatic, real-time, and continuous data collection, transmission, analysis, display, alarm, and other functions, which is convenient for managers to grasp the data of various ecological elements in the tourist area in real-time. 
Features of ecological environment monitoring subsystem: The host displays the collected data in realtime, and can remotely set the data collection time, storage and sending time interval, and other information. Modular design, the sensor can be configured arbitrarily through the host menu, a total of 16 types of sensors can be connected, and each sensor can be connected to 16 channels. With GPS function: The specific geographic location of the equipment and data collection point can be known through GPS, preventing theft and displacement. Equipped with a high-definition camera, which can take real-time photos and upload the scene to the platform.

\subsection{Pest Monitoring Subsystem}

The disease and insect pest monitoring and early warning subsystem integrate data collection, monitoring, expert system and other functions, intelligent monitoring, real-time collection of pest status information in the monitoring area. Remote diagnosis of pests, provide forest pest early warning information, improved the automation and rapidness of pest prevention and control, and achieved the requirements of rapid pest positioning, rapid deployment, command, and convenient, accurate, and intuitive management [20]. The equipment parameters of the pest monitoring subsystem are shown in Table 2.

Table 2. Equipment parameters of the pest monitoring subsystem

\begin{tabular}{|l|l|}
\hline Power supply & AC $220 \mathrm{~V} \pm 10 \%$ \\
\hline Attraction light source & $\begin{array}{l}\text { 20W insect trap lamp; } \\
\text { The dominant wavelength of } 365 \mathrm{~nm} ; \\
\text { Three impact screens form an angle of } 120^{\circ} \text { with } \\
\text { each other. }\end{array}$ \\
\hline Lamp start time & Less than 5 seconds after booting \\
\hline Insulation resistance & $>2.5 \mathrm{M} \Omega$ \\
\hline Camera & $500 \mathrm{~W} / 1200 \mathrm{~W}$ \\
\hline Display screen & 7 village capacitive touch screen \\
\hline System & Android4.0 \\
\hline
\end{tabular}

Features of this subsystem: The Android system intelligent control can be displayed and operated on the mobile phone. Built-in high-definition camera, can take pictures and upload regularly so that all-weather unattended automatic monitoring of wild insect information. Trapping principle: The ultraviolet trap lamp emits light that makes the pests sensitive, causing the pests to fly, hitting the glass screen, and falling to the insect-killing bin. Insect killing device: far-infrared heating treatment of pests, the lethality rate of the insects is not less than $98 \%$, and the integrity rate is not less than $95 \%$. Dried insects can be evenly scattered and spread on the conveyor belt without stacking. Light control: Automatically turn on the light at night and turn off the light automatically during the day (standby). The night working state, the working state will not be changed by momentary strong light. Time controlled: Set the working period according to the living habits of the target pests. Rain control: It can automatically control the work of the equipment according to changes in the outside weather (default work). Large and small bug identification filtering: The catching port is equipped with a filter to prevent non-target large bugs from entering the inside of the instrument and affecting the automatic identification of small bugs. Insect rain bin structure: rainwater is automatically drained, which can effectively separate rain and insects so that there is no water in the box.

\subsection{Big Data Intelligent Management Platform}

The big data integrated intelligent management platform gathers the information collected by each monitoring front end. Establish a comprehensive networked joint control information resource database and a comprehensive application system for information management. Form big data integrated intelligent management platform as the brain. The dual-spectrum thermal imaging camera is the eye. Each front sensor is a tentacle. The wireless ad hoc network is an integrated comprehensive information application system of the nerve center. With big data intelligent analysis services, expert online support, data release and sharing, equipment management, monitoring real-time preview, monitoring information management, early warning information management, energy security management, network management, electronic maps, largecapacity data storage, intelligent operation, and maintenance management, User management, multi-level platform management, and many other system functions. The system architecture of the big data integrated intelligent management platform is shown in Fig. 8. 


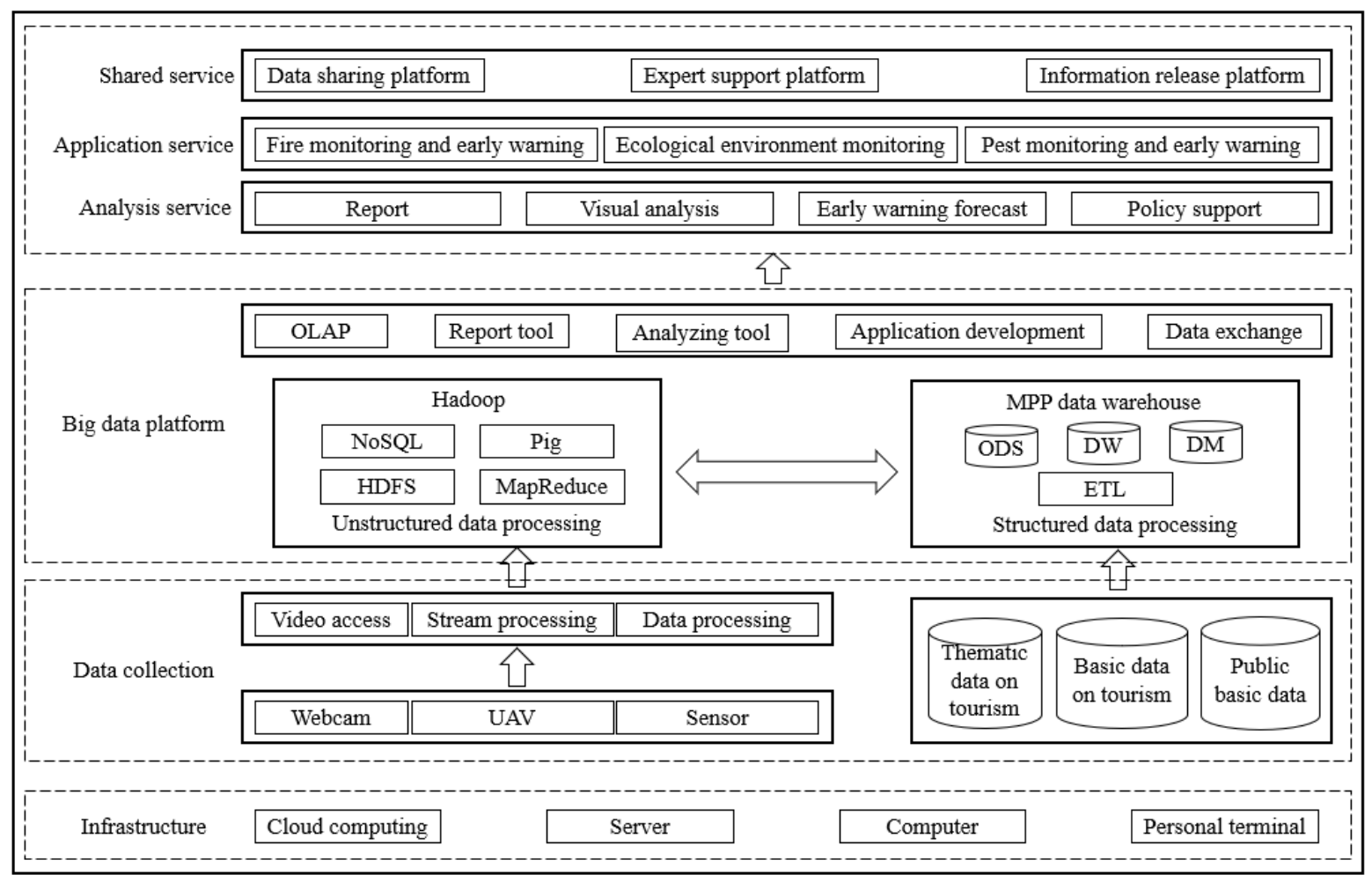

Fig. 8: The system framework of big data integrated intelligent management platform

Based on the big data storage system and distributed computing framework, it stores and manages multisource and multi-form data such as tourism industry thematic data, tourism basic data, and public basic data. Use the latest data mining technology and data visualization technology to fully reveal the regularity and value of data. Combining the expert support platform, data sharing platform, and information release platform, it provides strong information support and decision support for tourism management services such as fire monitoring and early warning, ecological environment monitoring, and pest monitoring and early warning. Big data integrated intelligent management platform is the core component of the construction of the smart tourism industry.

Functions of big data integrated intelligent management platform: User Management: Users can be added, deleted, and modified, user types can be set, and user rights can be managed to ensure the safe and effective operation of the platform. Real-time monitoring: Through the real-time monitoring function, the allweather and all-around video monitoring function of the monitoring network is realized. Real-time, intuitive, and clear monitoring of the monitoring target, the monitoring status of the front-end site can be observed 24 hours a day. Digital map: Provide electronic map function, integrated network management can view the attribute information of the device. Can connect according to each point, intuitively reflect the current network routing topology and network quality. Support to open real-time video, display alarm prompts on the map. Information management: Users can query alarm information, stored data, audio, and video data through the integrated management platform. And the system has the function of recording the user's operational information and providing the query function, which can trace the user's operation. Energy management: It can monitor the power generation status of the power supply system and the status of the energy storage system in real-time, and form a solid data foundation through high-precision and comprehensive data collection and real-time reporting. After big data and multi-dimensional analysis, the final analysis results are accurately presented in the form of reports. Intelligent analysis: Be able to analyze the historical fire location, cause of the fire, weather, tree species, topography, forest fire process, and other data in tourist areas. Predict the risk of fire in tourist areas and the prone areas to effectively prevent fire.

\section{Summary and Outlook}

The following functions can be achieved through the monitoring and management system designed for the southwest tourist area: (1) Real-time monitoring of tourist areas: Using a dual-spectrum thermal imaging 
camera, the visible light part can display high-definition color images during the day. Infrared thermal imaging can provide sharp and clear thermal images. Achieve continuous 24-hour day and night monitoring. (2) Ecological environmental monitoring: Real-time monitoring of soil, hydrology, water quality, air quality, and other information in the tourist area is carried out through the sensor of ecological elements in the tourist area. (3) Weather data collection: It can automatically monitor wind direction, wind speed, temperature, humidity, rainfall, etc. The on-site weather and image parameters can be transmitted to the command center every hour, and the fire risk level of the tourist area at that time can be automatically calculated. (4) Wireless communication network coverage in the monitoring area: The wireless ad hoc network is used to complete the network coverage of the tourist area without resorting to the operator's network and other fixed facilities. At the same time, the network can be connected to the command center and the public network through a variety of heterogeneous connection methods. (5) Analysis of intelligent big data platform: Through the intelligent analysis and diagnosis of the massive monitoring data collected and stored in the tourist area, the intrinsic value of the data is deeply excavated and converted into various effective information. (6) Intelligent fire hazard monitoring: According to the key fire hazard parts obtained by big data analysis, key tracking and monitoring are carried out. Realize unattended uninterrupted work, with the functions of automatically identifying fire points, reporting fire points in time, and accurately locating fire points. (7) Monitoring of diseases and pests in smart tourist areas: Real-time collection and monitoring of pest status information. Through the big data platform, the automation, rapidness, and intelligence of pest prevention and control are realized, and the requirements of rapid positioning, rapid deployment, and convenient, accurate, and intuitive management of pests and diseases are achieved. (8) Intelligent UAV cruise scheduling: UAV has the characteristics of fast maneuvering, low cost of use, high operating efficiency, large inspection area, and simple maintenance and operation. It has its unique advantages in monitoring areas that are not easily accessible to cars and people.

In summary, this system can realize the real-time collection, transmission, display, storage, and analysis of ecological environment information, and the low-cost, flexible and convenient layout can meet the needs of tourism area monitoring and management. The system can provide data support and technical support for the development of the southwest tourist area. The multi-subsystem design makes the system have good scalability and stability, can be popularized and used, and has a good application prospect.

\section{Acknowledgements}

This research has been aided in part by "This research was supported by the National Key Research and Development Project of China" (No. 2016YFC0502106) and "Study on ecological compensation mechanism of water quality and quantity in Yongding River Basin” (No. 2018ZX07111-002-006) which is supported by Major national science and technology projects of China.

\section{References}

[1] H. Xu, L. Zhai, S. Zhang. The Current Situation, Problems and Suggestions of China's Tourism Development. Foreign Economic Relations Trade. Chi. 2020(06):102-105.

[2] J. Liang, G. Yang, L. Wang. Eco-environment Monitoring Index and Monitoring Methods in the Ecotourism Area. Journal of Guangxi Normal University: Philosophy and Social Sciences Edition. Chi. 2008(03):57-60.

[3] J. Wu. Design and Implementation of People-Stream Surveillance and Statistic in Tourism. Dalian University of Technology. Chi. 2008.

[4] X. Wang. Ecological Monitoring and Health Assessment of the Source Water Quality in Yabuli Skiing Tourism Resort. Northeast Forestry University. Chi. 2012.

[5] W. Shi. Analysis and Influence Factors on Negative Oxygen Ion Monitoring in Chongming Scenic Area. The Administration and Technique of Environmental Monitoring. Chi. 2014,26(04):65-67.

[6] J. Chen, T. Yu, Y. Ju, Y. Chen, J. Shao. Biological Monitoring and Analysis of Water Quality of Water Supply Project in Yabuli Ski Tourist Area. Northern Chinese Fisheries. Chi. 2017(04):33-38.

[7] M. Lan. Ecology Health Assessment And Monitoring of Lushan Tourism Based on DPSIR Model. Jiangxi Normal University. Chi. 2018. 
[8] W. Lin. Monitoring and Protection of Forest Ecological Tourism Resources by Dynamic Monitoring System. Ecological Chemistry and Engineering. Eng. 2019, 26(1):189-197.

[9] Y. Huang, E. Hui, X. Yuan, J. Li. Sustainable exploitation and utilization of water resources in Southwest China. Journal of Water Resources \& Water Engineering. Chi. 2005, (02): 46-9+54.

[10] X. Shi, L. Zhang, J. Zhang, Z. Yang, Y. Xiao. Priority Area of Biodiversity Conservation in Southwest China. Chinese Journal of Ecology. Chi. 2018, 37(12): 3721-3728.

[11] X. Wu, L. Liu, Y. Sun. Ecological security evaluation based on entropy matter-element model: A case study of Kunming city, southwest China. Ecological Indicators. Eng. 2019, (102): 469-478.

[12] J. Xu. On the Evaluation Method of Jiuzhaigou Ecotourism Environmental Carrying Capacity. Technology and Economic Guide. Chi. 2018,26(12):75-76.

[13] H. Wang, L. Wu, Y. Wu. Research on the Application of Wireless Ad Hoc Network Technology in Emergency Communication Guarantee. Technology Discussion. Chi. 2013(02):41-44.

[14] A. Ito, T. Kai. A Study on Ad-hoc Network with BLE Advertisement. International Journal of Networking and Computing. Eng. 2020, 10(2):94-110.

[15] P. Xue, J. Wu, P. Rong. Evaluation of Information Brittleness Risk in Network Communication System. Electric Machines and Control. Chi. 2010(08):75-80.

[16] Q. Liu, X. Ma. Security Model and Design of Network Communication System Based on Data Encryption Algorithm. International Journal of Autonomous and Adaptive Communications Systems.Eng. 2021, 14(2):1.

[17] X. Yu, X. Li. Application Analysis of Water Quality Automatic Monitoring and Early Warning System in Hongshan Park of Chifeng City. China Resources Comprehensive Utilization. Chi. 2021,39(01):59-61.

[18] P. Xue, A. Yun. Design of GIS-based Monitoring and Early-warning System of Landslide Hazard in Diao Zhongba - ScienceDirect. Energy Procedia. Eng. 2012, (16):1174-1179.

[19] L. Shu, W. Yan. Daily Security of System Safety of Power and Energy Safeguard Department of Wuhan Tianhe Airport. Journal of Green Science and Technology. Chi. 2011(09):127-128.

[20] S. Yang. Risk Management of Intelligent Pest Monitoring System Project Development. Nanjing University of Posts and Telecommunications. Chi. 2020. 